\title{
Gestión de la vida recreativa: ¿Un factor de riesgo determinante en el uso reciente de drogas?
}

\author{
Amador Calafat; Cesáreo Fernández Gómez; Montse Juan; Elisardo Becoña \\ Irefrea - España. \\ Enviar correspondencia a: \\ Amador Calafat, Rambla, 15, 2 - $3^{\mathrm{a}} .07003$ Palma de Mallorca (España) tel. +34971727434 Fax: 971213306 irefrea@irefrea.org \\ Recibido: 15 de octubre de 2004 \\ Aceptado: 10 de mayo de 2005.
}

\section{RESUMEN}

El objetivo es describir los hábitos recreativos, uso de drogas y conductas de riesgo entre jóvenes que salen de noche durante los fines de semana, y estimar la relevancia predictiva de la vida recreativa sobre el uso de drogas legales o ilegales, teniendo en cuenta otros factores de riesgo individuales. La muestra incluye 1340 jóvenes entrevistados en cinco ciudades españolas. Casi todos abusan del tabaco y alcohol. Se ha dividido la muestra en los que consumieron $(775 ; 58 \%$ ) o no alguna droga ilegal recientemente $(565 ; 42 \%)$. El uso de drogas ilegales aparece asociado con diversas características personales: inicio más temprano en el consumo de cannabis y alcohol, sexo masculino, menor percepción del riesgo en el uso de drogas, mayor búsqueda de sensaciones y mayor implicación en conductas - problema y desviadas socialmente. Los consumidores de drogas ilegales se distinguen también en sus hábitos recreativos (salen más, buscan contextos con mayor disponibilidad de drogas, tienen distintas motivaciones para salir). Un análisis multivariante clasificó correctamente casi cuatro de cada cinco sujetos no-consumidores o consumidores de drogas ilegales. Entre los factores estudiados, los relacionados con la vida recreativa fueron los mejores predictores del uso de drogas. Se desprende de estos datos la necesidad de estudiar en profundidad la vida recreativa de los jóvenes e integrar el contexto recreativo en los objetivos de la prevención del uso y abuso de drogas.

Palabras clave: jóvenes, prevención, factores de riesgo, alcohol, drogas ilegales, actividades recreativas.

\begin{abstract}
The objective is to describe recreational habits, drug use and risk behaviours in the young going out for enjoyment the weekends, and to estimate the predictive relevance of recreational related variables on drug use, in comparison with other intrapersonal risk factors. The sample comprises 1340 young people interviewed in 5 Spanish cities. Almost all of them abused alcohol or tobacco recently. We divided the sample in two groups, using $(775 ; 58 \%)$ any illicit substance last month or not $(565 ; 42 \%)$. The use of illegal drugs is associated in our sample with a number of intrapersonal factors: younger ages of onset for cannabis and alcohol, male sex, lower risk perception, greater sensation seeking, involvement in risk behaviours and in problem behaviours. Illegal drug users are also distinguished by their recreational habits (greater involvement and different motivation in going out looking for different contexts in recreational nightlife). A multivariate analysis correctly classified four out of five individuals using legal or illegal drugs. Among the factors studied, recreational styles were the best predictors of drug use. It suggests the need to make an in depth study of weekend recreational habits and add them to the objectives of the prevention of drug use and misuse.
\end{abstract}

Keywords: youth, prevention, risk factors, alcohol, illegal drugs, recreational activities.

\section{INTRODUCCIÓN}

os informes periódicos de ámbito internacional (Observatorio Europeo sobre Drogas y Toxicomanías, Naciones Unidas, etc.) indican que los problemas relacionados con el consumo de drogas aumentan en la mayoría de los países europeos ${ }^{1-3}$. Aunque la prevención del uso de drogas ha demostrado ser efectiva cuando está científicamente fundamentada y basada en la evidencia ${ }^{4}$, las inversiones en prevención e investigación orientada a la preven- ción son reducidas y una mayoría de las actuaciones preventivas no se basan en los avances en el conocimiento alcanzados en este área. Se reconoce la necesidad de comprender mejor la naturaleza e interacción entre los factores de riesgo y protección en el uso de drogas, como una base para el desarrollo de modelos predictivos e intervenciones preventivas bien fundamentadas ${ }^{5,6}$.

El estudio de los factores de riesgo y protección y el desarrollo de modelos teóricos en el uso de drogas 
está relativamente bien establecido en Norteaméri$\mathrm{ca}^{6,10}$. En una revisión reciente sobre estos desarro$\|{ }^{11}$, se observan limitaciones muy sustanciales en la investigación Europea en este ámbito: a) Falta de estudios que integren factores ambientales relevantes y exploren su relevancia relativa en el uso y abuso de drogas; b) Escasez notable de estudios que se enfoquen en el abuso de drogas, más allá de estudios sobre prevalencia del uso de drogas. Los factores de riesgo señalados como mejores predictores en el uso problemático de drogas han sido clasificados en: a) Intrapersonales: Personalidad - Rasgos Psicológicos, Vulnerabilidad y Conducta Problema y edad de inicio temprana en el consumo de drogas; b) Micro-ambientales: Familia y uso de drogas, estructura y calidad en las relaciones, influencia de los pares; y c) Macroambientales: Deprivación social y económica, Difusión social de culturas relacionadas con las drogas (normalización del consumo de drogas).

En los estudios y revisiones sobre los factores de riesgo y protección que influyen en el uso de drogas, los hábitos recreativos y la diversión no suelen ser tomados en consideración como determinantes del uso y abuso de drogas, con algunas excepciones ${ }^{12}$. La vida recreativa del fin de semana ha experimentado un desarrollo muy sustancial entre los jóvenes durante las dos últimas décadas, y se ha establecido en áreas donde música, modas y el uso de drogas legales e ilegales se han convertido en elementos esenciales de la diversión y su contexto. Las drogas actúan en estos espacios como estimulantes, desinhibidores y elementos de cohesión que facilitan el logro de los objetivos de la diversión ${ }^{13}$. Durante los últimos 9 años Irefrea ha venido desarrollando estudios que combinan técnicas de investigación cualitativas y encuestas en España y otros países Europeos ${ }^{14,18,19}$ con el fin de comprender mejor los nuevos patrones de consumo de drogas y sus determinantes. El objetivo general de este informe es describir los jóvenes Españoles que salen frecuentemente a divertirse en los espacios del fin de semana en diferentes ciudades, analizar su consumo de alcohol y drogas e identificar las relaciones entre su uso de drogas y los factores de riesgo y protección individuales más relevantes según la literatura especializada. Los objetivos específicos son:

1. Describir los jóvenes usuarios de los espacios recreativos del fin de semana en cinco ciudades Españolas y comparar dos grupos de ellos: quienes usan / abusan de drogas legales (alcohol y tabaco) y quienes, además de usar drogas legales también consumen en el momento del estudio una o más drogas ilegales.

2. Identificar los factores de riesgo y protección individuales que predicen el uso y abuso de drogas legales e ilegales, teniendo en cuenta los principales factores de este nivel intrapersonal y la gestión que estos jóvenes realizan de su vida recreativa nocturna durante los fines de semana.

3. Estimar la capacidad predictiva relativa de estos factores de riesgo y protección en el uso y abuso de drogas e identificar los de mayor relevancia para el uso y abuso de drogas en los contextos recreativos.

\section{MATERIAL Y MÉTODO}

\section{Descripción de la muestra}

La población objeto de estudio está formada por los jóvenes que participan en la vida recreativa nocturna durante los fines de semana en varias ciudades Españolas. De esa población han sido seleccionados y entrevistados un total de 1340 adolescentes y adultos jóvenes, participantes de las cuatro subculturas recreativas más significativas en cinco ciudades: Bilbao, Madrid, Palma de Mallorca, Valencia y Vigo. Antes de realizarse este estudio de encuestas, con el fin de identificar las subculturas y contextos recreativos clave en la vida nocturna donde se desarrolla la diversión de los jóvenes durante los fines de semana. Se utilizó un procedimiento para seleccionar de un modo aproximadamente aleatorio individuos entre los usuarios de estos espacios recreativos de cada ciudad. Una descripción detallada de los procedimientos para la selección de espacios recreativos y sujetos participantes pueden hallarse en otros informes ${ }^{15,19}$. El estudio de encuestas fue desarrollado en el periodo de otoño - invierno 1998-1999.

La muestra total ( $N=1340$ ) está formada por más varones $(60,9 \%)$ que mujeres $(39,1 \%)$, con una edad promedio de 22,6 años (desviación estándar = 5 años), casi todos solteros (92\%), con alguna actividad laboral (54\%) o dedicados a los estudios (41\%), habiendo cursado estudios secundarios (43\%) o universitarios (43\%), conviviendo con la familia de origen $(77 \%)$ de estatus socioeconómico medio (54\%) o superior (34\%) que ejercía o ejerce un control nulo $(25 \%)$ o escaso (45\%) sobre la vida recreativa de estos jóvenes. Por lo tanto, estos adolescentes y adultos jóvenes distan mucho de ser jóvenes en situación desfavorecida o de marginalidad, como se encuentra habitualmente entre colectivos de usuarios de drogas.

\section{Instrumento}

Los individuos participantes en el estudio fueron entrevistados utilizando un cuestionario estructurado 
formado por 50 cuestiones relativas a características sociodemográficas, consumo de drogas, conductas orientadas a la búsqueda de sensaciones, conductas de riesgo y socialmente desviadas y hábitos recreativos durante las noches del fin de semana. Las respuestas a los ítems sobre los hábitos recreativos fueron seleccionadas entre los contenidos más relevantes elicitados durante el estudio cualitativo previo. Las variables objeto de estudio en este informe son:

1. Características sociodemográficas: edad, sexo, estado civil, nivel educativo, autovaloración como estudiante, lugar donde vive, ocupación, estatus socioeconómico familiar percibido, control familiar sobre los hábitos recreativos nocturnos del fin de semana, edad de inicio en el consume de drogas legales e ilegales.

2. Hábitos y opiniones relativas a la vida recreativa nocturna del fin de semana: Indicadores de implicación en la vida recreativa nocturna (tiempo y recursos económicos empleados), contexto cultural y físico donde se desarrolla y significado que adquiere para el individuo. Se incluyen cuestiones sobre la frecuencia de salir a diferentes lugares (bares, discos, pubs, cafés, after hours, raves, etc.), frecuencia de salir cada mes y cada fin de semana, duración de una sesión típica, número de lugares visitados, hábito de recorrer grandes distancias, razones para elegir un lugar (7 ítems), y razones para salir de marcha (7 ítems).

3. Frecuencia de consumo de diferentes substancias y percepción del riesgo asociado al consumo de drogas: alcohol, tabaco, cannabis, LSD, éxtasis, cocaína, speed.

\section{Búsqueda de sensaciones: (3 ítems).}

5. Conductas de riesgo y desviadas socialmente: Cinco ítems relativos a conducir bajo efectos de alcohol o drogas ilegales y otras cuatro conductas (conducir sin licencia, vandalismo, hurtos y violencia).

\section{Procedimientos}

Con el fin de comparar dos grupos de consumidores de drogas e identificar los factores individuales de riesgo para el consumo de drogas se presentan descriptivos básicos (frecuencias, porcentajes, valores promedio y dispersión) y se utilizan contrastes paramétricos (ANOVA) y no paramétricos (Chi cuadrado con pruebas exactas de significación bilateral). Para estimar la capacidad predictiva relativa sobre el consumo de drogas se utilizaron análisis de regresión logística multinomial. Los análisis estadísticos fueron realizados con el paquete estadístico SPSS para Windows.

\section{RESULTADOS}

Teniendo en cuenta que la muestra está compuesta por jóvenes socialmente integrados y relativamente normativos, las frecuencias de consumo y abuso de alcohol y otras drogas son altas. Las prevalencias de consumo halladas son más altas que las informadas en la población general de edades similares. Casi todos han probado el alcohol $(98,8 \%)$ y el tabaco $(82,1 \%)$, una gran mayoría han probado el cannabis $(75,5 \%)$ y porcentajes sustanciales han Ilegado a consumir alguna vez éxtasis $(37,5 \%)$, cocaína $(42,4 \%)$, anfetaminas $(29,2 \%)$ y LSD $(38,3 \%)$. Casi todos consumen alcohol semanalmente $(81,3 \%)$, se han embriagado más de una vez durante el último mes (84\%) y una mayoría fuman diariamente $(61,6 \%)$. Durante el último mes, más de la mitad habían consumido cannabis (53\%), un porcentaje sustancial aunque menor había consumido éxtasis (13\%), cocaína (17\%), anfetaminas (7\%) o LSD (7\%) y una mayoría (60\%) habían abusado de dos drogas ilegales (fumando diariamente y emborrachándose durante este periodo).

La mitad de la muestra ha conducido en alguna ocasión bajo los efectos del alcohol (51\%) y más de un tercio han conducido vehículos bajo los efectos de alguna droga ilegal (37\%), una mayoría (63\%) continúan consumiendo alguna sustancia que les ha llegado a ocasionar problemas y casi la mitad han recibido avisos de familiares o amigos que perciben un claro abuso de drogas (40\%). En resumen, el perfil prototípico de la muestra se define por un abuso del alcohol, tabaco y cannabis, y una presencia sustancial del éxtasis, cocaína, LSD y anfetaminas. Nos encontramos de este modo bastante alejados del mito de que los consumidores recreativos de drogas eran principalmente consumidores de éxtasis que evitaban el abuso de otras drogas (especialmente el alcohol). Este patrón de consumo de drogas predominante se caracteriza por un policonsumo y uso problemático de diferentes sustancias ${ }^{20}$.

\section{Dos patrones de consumo de drogas en espacios recreativos}

En esta muestra, los individuos que no abusan de drogas legales (fuman diariamente o se embriagan repetidamente cada mes) ni consumen ninguna droga ilegal en el último mes forman un grupo relativamente marginal (23 sujetos, 4,1\%). Los pocos sujetos que no se habían embriagado durante el mes anterior fuman diariamente tabaco y han consumido cannabis durante el mes anterior. Por tanto, no encontramos sujetos que puedan ser considerados no-consumidores de drogas (ni legales, ni ilegales). 
Dividimos la muestra en dos subgrupos, según el consumo de tabaco, alcohol y drogas ilegales:

1) Un individuo es denominado consumidor de drogas legales si no ha llegado a consumir drogas ilegales durante el último mes. En esta muestra, 565 (42\%) mantienen este patrón de consumo en el mes previo a la encuesta.

2) Un individuo es denominado consumidor de drogas ilegales (cannabis, éxtasis, anfetaminas, cocaína, LSD, hongos, opiáceos) cuando ha consumido alguna de ellas durante el último mes previo a la encuesta, llegando a ser mayoría entre los sujetos de la muestra (775; 58\%). Además, casi todos ellos se han embriagado durante el último mes (99\%) y fuman diariamente (75\%), un patrón de consumo algo más frecuente de lo hallado en otros estudios europeos sobre consumo recreativo de drogas ${ }^{21}$.

\section{Capacidad predictiva de las características socio- demográficas, gestión de la vida recreativa y acti- tudes relativas a los riesgos sobre el consumo de drogas}

Una vez definidos estos dos patrones de consumo de drogas en espacios recreativos, nos interesa estimar la capacidad predictiva de diferentes grupos de variables sobre estos consumos de drogas. Hemos sometido a estudio tres grupos de variables: a) sociodemográficas y relativas a la edad de inicio en el consumo de diferentes sustancias; b) gestión de la vida recreativa y c) conductas de riesgo, conductas - problema o desviadas, búsqueda de sensaciones y percepción de los riesgos asociados al consumo de drogas. En una primera fase identificamos las variables predictoras (asociadas con estos patrones de consumo) dentro de cada grupo y su capacidad conjunta para clasificar a los individuos en su patrón de consumo. En un paso posterior, incluimos en el análisis multivariante los mejores predictores de cada grupo de variables para estimar su relevancia predictiva relativa y la capacidad global para clasificar a los sujetos en su patrón de consumo de drogas.

\section{Capacidad predictiva de las variables sociodemo- gráficas en el consumo de drogas}

Los consumidores de drogas ilegales son mayoritariamente varones (68\%), con estudios secundarios $(44 \%)$ o inferiores $(17 \%)$ en los que se autovaloran como estudiantes regulares o malos ( $45 \%$ y $24 \%$ respectivamente), están trabajando (39\%) o simultanean estudios y trabajo (19\%), conviven con la familia de origen $(70 \%)$ de nivel socioeconómico medio o más bajo $(67 \%)$ que ejerce o ha ejercido un control nulo o escaso sobre su vida recreativa (77\%). Tienen una edad promedio de 23,1 años (1,4 años más que los consumidores sólo de drogas legales), se iniciaron en el consumo de alcohol a una edad promedio de 14,2 años (casi un año en promedio antes que el otro grupo), en el consumo de tabaco a una edad promedio de 14,5 años (un año en promedio antes que los consumidores de drogas legales que han llegado a probarlo) y en el cannabis a una edad promedio de 15,5 años (1,3 años en promedio antes que los consumidores de drogas legales que han llegado a consumirlo).

Los consumidores que usan / abusan frecuentemente de drogas legales sin consumir recientemente drogas ilegales son casi por igual varones (51\%) y mujeres (49\%), con estudios universitarios (50\%) o secundarios (42\%) en los que se autovaloran como estudiantes buenos (49\%) o regulares (41), y están estudiando $(50 \%)$ en ocasiones de modo simultáneamente con un trabajo (18\%). Conviven con una familia de origen $(86 \%)$ de nivel socioeconómico medio $(58 \%)$ o superior $(35 \%)$ que ejerce o ha ejercido un control moderado sobre su vida recreativa. Tienen una edad promedio de 21,8 años.

El sexo masculino se asocia significativamente con el uso de drogas ilegales $\left(\mathrm{Chi}^{2}=36,9\right.$; p exacta $<0,001)$. Probablemente, las diferencias en socialización, rol y supervisión (entre otros aspectos) están en la base de estas diferencias entre géneros. Se observa una edad promedio significativamente menor entre los consumidores solo de drogas legales que entre los consumidores de drogas ilegales y un inicio significativamente más tardío en el consumo de alcohol, tabaco y cannabis ( $p<0,001$ en el ANOVA para las cuatro variables). El alcanzar los estudios universitarios se asocia significativamente con ser consumidor solo de drogas legales, mientras que haber completado solamente los estudios primarios se asocia significativamente con ser consumidor de drogas ilegales $\left(\mathrm{Chi}^{2}=\right.$ 28,4; p exacta $<0,001$ ). De modo similar, el valorar que uno ha sido un mal estudiante se asocia con ser consumidor de drogas ilegales mientras que haber sido un buen estudiante se asocia significativamente con ser consumidor solo de drogas legales $\left(\mathrm{Chi}^{2}=\right.$ 59,3; p exacta <0,001). El proseguir con los estudios se asocia con el consumo solamente de drogas legales, mientras que estar empleado se asocia significativamente con el consumo de drogas ilegales en estos usuarios de los espacios recreativos $\left(\mathrm{Chi}^{2}=38,6 ; \mathrm{p}\right.$ exacta $<0,001)$. Vivir con la familia de origen se asocia con el consumo solo de drogas legales, mientras que vivir con amigos o solo se asocia significativamente con el consumo también de drogas ilegales $\left(\mathrm{Chi}^{2}=\right.$ 46,2; p exacta $<0,001)$. Los consumidores solamente de drogas legales e ilegales tienen una probabilidad mayor de la esperada de proceder de familias con un estatus medio-bajo $\left(\mathrm{Chi}^{2}=22,8\right.$; p exacta $\left.<0,001\right)$ 
que han ejercido o ejercen un nulo control sobre sus hábitos recreativos $\left(\mathrm{Chi}^{2}=45,2 ;\right.$ p exacta $\left.<0,001\right)$.

\section{Predicción del uso de drogas basada en la regre- sión logística con variables sociodemográficas}

El análisis de regresión logística resultó en una ecuación que alcanzó un ajuste adecuado (Chi cuadrado $=572,5 ; p<0,001$ para 6 grados de libertad). El modelo clasificó correctamente al $77 \%$ de la muestra en su grupo de consumo, con una capacidad muy alta para clasificar a los consumidores de alguna droga ilegal $(92 \%)$ y una capacidad moderada para los consumidores de drogas legales (57\%). De los factores pertenecientes a este dominio de variables que están asociados al consumo de drogas, 6 fueron incluidos en la ecuación de regresión. El factor de riesgo más relevante fue el haber llegado a probar el cannabis, con una gran diferencia sobre los demás, hasta multiplicar por 53 las probabilidades de ser consumidor de alguna droga ilegal en comparación con los que no han llegado a probarlo. Vivir en lugar distinto a la familia de origen o pareja casi triplica la probabilidad de ser consumidor de alguna droga ilegal, seguida de haber sido un mal estudiante (duplica las probabilidades) y ser varón y proceder de una familia con nulo control sobre el salir de marcha (en ambos casos, se aumenta en un $50 \%$ las probabilidades de ser consumidor de alguna droga ilegal). El haber probado el tabaco ya con una edad superior a los 15 años resulta ser un factor de protección (disminuye a la mitad las probabilidades de ser consumidor de alguna droga ilegal).

\section{Capacidad de los hábitos, intereses y motivacio- nes recreativas para predecir el uso de drogas}

Los contextos donde se desarrolla la vida recreativa nocturna están entre los principales espacios para la socialización de los jóvenes, encontrarse con los amigos, integrarse en un grupo de pares y amigos y desarrollar unas relaciones basadas en unos valores y normas diferentes de las que regulan las relaciones familiares, educativas, laborales, etc. El concepto de vida recreativa nocturna del fin de semana se refiere a un fenómeno relativamente reciente, de emergencia en las últimas dos décadas.

Como se describe en la tabla 1, estos jóvenes usuarios de los espacios recreativos del fin de semana salen con unas motivaciones principales: escapar de la rutina, escuchar música, reunirse con los amigos y bailar. La duración típica de una sesión podría decirse que está entre las 7 y las 9 horas, la mayoría de estos jóvenes salen de marcha todos o casi todos los fines de semana y lo hacen durante más de una noche cada fin de semana. Los lugares frecuentados habitualmente son bares y pubs, y en menor frecuen- cia discos y cafés. Los criterios principales en que se basan estos jóvenes para elegir un local recreativo durante el fin de semana son su ambiente (atmósfera), la música y clientela característicos del mismo, y en menor medida sus precios.

Más allá de estas características generales en la vida recreativa del fin de semana, se observan diferencias estadísticamente significativas y más o menos sustanciales en la implicación, significado y contexto de estos hábitos entre los que consumen drogas legales o ilegales.

1. En lo relativo a la implicación en el salir de marcha: Los consumidores solo de drogas legales tienen una probabilidad significativamente mayor que los que consumen alguna droga ilegal de salir solamente uno o dos fines de semana al mes (Chi cuadrado = 7,4; p exacta $<0,05$ para 2 grados de libertad), salir una sola noche cada fin de semana (Chi cuadrado = 28,9; p exacta $<0,001$ para 2 g.l.), salir un número de horas promedio significativamente menor en una sesión típica ( $F=108,4 ; p<0,001$ para 1 g.l.), menor probabilidad de salir durante los días laborables (Chi cuadrado $=74,7 ;$ p exacta $<0,001$ para 2 g.l.) y menor probabilidad de realizar largos desplazamientos cuando salen de marcha (Chi cuadrado $=45,3 ;$ p exacta $<$ 0,001 para 2 g.l.).

2. En cuanto al contexto donde se desarrolla la vida recreativa nocturna: Los consumidores de alguna droga ilegal tienen una probabilidad significativamente mayor que los que solo consumen drogas legales de ir frecuente o muy frecuentemente a bares (Chi cuadrado = 26; $p$ exacta $<0,001$ para 3 g.l.), "After hours" (Chi cuadrado $=171,7 ;$ p exacta $<0,001$ para 3 g.l.) y fiestas "Rave" (Chi cuadrado $=133$; $p$ exacta $<0,001$ para 3 g.l.). Las diferentes subculturas recreativas también se asocian significativamente con el patrón de consumo de drogas: Los consumidores de droga silegales tienen mayor probabilidad de integrarse en grupos y culturas "After tour" o "Alternativos" (Chi cuadrado $=136,8 ;$ p exacta $<0,001$ para 3 g.l.). Algunas características específicas de los contextos recreativos también marcan diferencias significativas entre unos y otros. A la hora de elegir un lugar, los consumidores solo de drogas legales tienen mayor probabilidad de considerar importante o muy importante los precios del local (Chi cuadrado $=27,5 ; \mathrm{p}$ exacta $<0,001$ para 3 g.l.), mientras que los consumidores de drogas ilegales dan mayor importancia que los primeros a la posibilidad de encontrar pareja sexual (Chi cuadrado $=10,9$; $p$ exacta $<0,05$ para 3 g.l.), pero principalmente a la disponibilidad de drogas (Chi cuadrado $=368,9$; p exacta $<0,001$ para 3 g.l.).

3. También los motivos (significado) para salir son distintos en algunos aspectos entre estos dos grupos de consumidores de drogas. Quienes consumen solo drogas legales dan mayor importancia que los que 
Tabla 1: Asociación entre salir de marcha y consumo de drogas: diferentes estilos recreativos (implicación, significado y contexto físico-cultural) en dos patrones de consumo.

\begin{tabular}{|c|c|c|c|c|}
\hline & \multicolumn{2}{|c|}{$\begin{array}{l}\text { Solo drogas legales } \\
\qquad(n=565)\end{array}$} & \multicolumn{2}{|c|}{$\begin{array}{c}\text { También drogas ilegales } \\
(\mathrm{n}=775)\end{array}$} \\
\hline & $\mathbf{N}$ & $\%$ & $\mathbf{N}$ & $\%$ \\
\hline \multicolumn{5}{|l|}{ Implicación en salir de marcha } \\
\hline Sale $3 / 4$ fines de semana al mes & 406 & 72 & 603 & 78 \\
\hline Sale solo una noche / fin de semana & 214 & 38 & 225 & 29 \\
\hline Sale durante los días laborables & 112 & 20 & 329 & 43 \\
\hline Duración de una sesión típica en horas (media, D.E.) & 6,8 & 2,2 & 9,4 & 5,6 \\
\hline Recorre largas distancias cuando sale normalmente & 106 & 19 & 276 & 36 \\
\hline \multicolumn{5}{|l|}{ Va a menudo o muy a menudo a } \\
\hline Bares & 335 & 59,3 & 556 & 71,8 \\
\hline Discos & 209 & 37,1 & 313 & 40,6 \\
\hline Pubs & 318 & 56,5 & 387 & 50,2 \\
\hline Cafés & 208 & 37,2 & 249 & 32,4 \\
\hline Afters & 32 & 5,8 & 195 & 25,3 \\
\hline Raves & 16 & 3 & 84 & 11 \\
\hline \multicolumn{5}{|l|}{ Razones para elegir un lugar (bar, pub, etc.) } \\
\hline Precios (importante) & 405 & 72,2 & 485 & 62,8 \\
\hline Atmósfera (importante) & 543 & 96,8 & 724 & 93,6 \\
\hline Música (importante) & 504 & 89,6 & 694 & 89,8 \\
\hline Tipo de clientela (importante) & 475 & 84,5 & 635 & 82,2 \\
\hline Distancia & 164 & 25,4 & 220 & 28,7 \\
\hline Posibilidad de encontrar pareja sexual & 210 & 37,3 & 329 & 42,7 \\
\hline Drogas (alguna importancia) & 128 & 22,8 & 570 & 73,9 \\
\hline \multicolumn{5}{|l|}{ Razones para salir de marcha } \\
\hline Reunirse con los amigos (muy importante) & 380 & 67,5 & 478 & 61,8 \\
\hline Escuchar música (importante) & 412 & 73,1 & 622 & 80,5 \\
\hline Escapar de la rutina (importante) & 465 & 83 & 612 & 79,9 \\
\hline Bailar (importante) & 341 & 60,6 & 398 & 51,7 \\
\hline Consumir drogas (alguna importancia) & 116 & 20,6 & 634 & 82,1 \\
\hline Buscar sexo (importante) & 172 & 30,6 & 349 & 45,2 \\
\hline Buscar pareja (importante) & 142 & 25,4 & 190 & 24,8 \\
\hline \multicolumn{5}{|l|}{ Subcultura recreativa de referencia } \\
\hline After - hour (tecno) & 69 & 12,2 & 194 & 25 \\
\hline Aternativos & 62 & 11 & 210 & 27,1 \\
\hline Adolescentes & 189 & 38,5 & 217 & 28 \\
\hline Normativos ("Mainstream") & 245 & 43,4 & 154 & 19,9 \\
\hline
\end{tabular}

consumen alguna droga ilegal a bailar (Chi cuadrado $=25,7$; $p$ exacta $<0,001$ para 3 g.l.) y encontrarse con los amigos (Chi cuadrado $=9,3 ;$ p exacta $<0,05$ para 3 g.l.). Inversamente, estos últimos le dan una importancia significativamente mayor que los primeros a escuchar música (Chi cuadrado $=24,8$; p exacta $<0,001$ para 3 g.l.), buscar sexo (Chi cuadrado $=46,6$; p exacta $<0,001$ para 2 g.l.) y especialmente a consumir drogas como motivo para salir (Chi cuadrado = 554; p exacta $<0,001$ para 3 g.I.).

\section{Regresión logística de los hábitos recreativos so-} bre el consumo de drogas legales o ilegales

El análisis de regresión logística multinomial resultó en una ecuación con un adecuado nivel de ajuste (Chi cuadrado $=700,1 ; p<0,001$ para 9 g.l.), y que clasificó correctamente a más de cuatro de cada cinco sujetos participantes en el estudio (83\%), con una capacidad clasificatoria algo superior para los consumidores de drogas ilegales (86\%) que para los consumidores de 
drogas legales (78\%). De todos los factores de este dominio de variables que estaban asociados con el consumo de alguna droga ilegal, el modelo basado en la regresión logística incluyó 9, relativos a los tres conceptos: implicación en la vida recreativa nocturna del fin de semana, contexto en que se desarrolla y significado que alcanza para el individuo. Los factores más relevantes resultaron ser relativos al significado y contexto: la nula importancia del consumo de drogas como motivador para salir (divide por 10 la probabilidad de ser consumidor de alguna droga ilegal) y para elegir un lugar (divide por 3 esta probabilidad). Otros factores protectores son relativos a una menor implicación (no salir durante los días laborables, salir en promedio durante menos de 8 horas por sesión). Al contrario, realizar largos desplazamientos para divertirse resulta ser un factor de riesgo. Otros factores relativos al significado incluidos en la ecuación fueron otorgar un valor moderado (no máximo) al escuchar música como motivo para salir como factor protector y no otorgar ninguna importancia al baile como motivo para salir (factor de riesgo). Otros dos factores protectores relativos al contexto resultaron ser no ir nunca a fiestas "rave" y tener como referencia subculturas recreativas cercanas a los más jóvenes y a jóvenes "normativos" (no a las culturas "after hour" o "alternativas".

Una buena parte de la investigación relativa a los hábitos recreativos y el consumo de drogas se refiere a los contextos rave y locales "after hour"22, 25. Nuestros resultados indican claramente que el consume de drogas ilegales está determinando en mayor medida por otros hábitos recreativos, aunque los contextos rave y tecno resulten también claramente relevantes. De modo similar a lo hallado en otros estudios, nuestros resultados sugieren que el enfoque preventivo no debería ser exclusiva ni preferentemente dirigido a estos contextos que tampoco son mayoritariamente utilizados por los usuarios de la vida recreativa, aunque muchos usuarios problemáticos de drogas pueden estar vinculados a estos contextos ${ }^{26}$.

Nuestros resultados apoyan un enfoque preventivo más amplio sobre determinadas subculturas recreativas que pueden ser factores de riesgo o protección de primer orden. Componentes de la mayor relevancia predictiva dentro de la vida recreativa son una implicación intensiva y dogmática con la vida recreativa nocturna y, especialmente, un significado de la diversión que integra el consumo de drogas como elemento esencial, asociado a la intensa estimulación sensorial (la experimentación intensa de la música).

\section{Capacidad predictiva de las percepciones del ries- go, conductas de riesgo, búsqueda de sensaciones y conducta desviada en el consumo de drogas}

Existe una relación positiva y bien documentada entre el consumo de drogas y una serie de conductas de riesgo (conducta sexual y otras prácticas de riesgo para la salud) y socialmente desviadas (conducción de vehículos bajo efectos de drogas y alcohol, violencia, etc.). También existe una amplia literatura sobre la asociación entre el consumo de drogas y rasgos de personalidad relacionados con la búsqueda de sensaciones, impulsividad, etc., baja percepción del riesgo asociado al consumo de drogas, etc. En nuestra muestra, los consumidores de alguna droga ilegal también presentan un perfil diferenciado en estas características relacionadas con los riesgos. La mayoría (58\%) de los consumidores de alguna droga ilegal habían conducido vehículos bajo los efectos del alcohol (40\% entre el otro grupo de consumidores), y también bajo el efecto de drogas ilegales (57\%, en comparación del $9 \%$ en el otro grupo de consumidores). La gran mayoría de los consumidores de alguna droga ilegal se habían implicado tempranamente (antes de los 15 años) o recientemente (en el último año) en una o más de las conductas desviadas evaluadas: conducir sin licencia $(40,2 \% ; 24 \%$ en el otro grupo), destrozos y vandalismo (37,7\%; 13,3\% en el otro grupo), hurtos en tiendas y almacenes $(68,3 \% ; 35,2 \%$ en el otro grupo) y peleas fuera del entorno familiar (52\%; 40\% en el otro grupo). Alrededor de uno de cada cuatro en ambos grupos perciben un riesgo elevado en fumar un paquete de cigarrillos diariamente; son menos aún los que perciben este riesgo en consumir dos bebidas alcohólicas diariamente o cuatro bebidas alcohólicas seguidas. Menos de un tercio $(27 \%)$ de los consumidores de alguna droga ilegal perciben un riesgo al menos moderado en el consumo regular de cannabis (67\% en el otro grupo), y algo más de la mitad perciben un riesgo alto en consumir habitualmente éxtasis cada fin de semana (55\%; 83\% en el otro grupo).

La frecuencia de las cuatro conductas desviadas evaluadas fue significativamente mayor en el grupo consumidor de alguna droga ilegal (Chi cuadrado exacto < 0,001 en las cuatro), y también en las dos conductas de riesgo en la conducción de vehículos: (Chi cuadrado $=39,3$; $p$ exacto $<0,001$ para la conducción bajo efectos del alcohol y Chi cuadrado $=323,2$; p exacto $<0,001$ para la conducción bajo efectos de otras drogas). También lo es la frecuencia de los tres ítems sobre búsqueda de sensaciones evaluados: hacer lo que le apetece, sea lo que fuere; hacer cosas peligrosas en respuesta a desafíos o hacer cosas locas aunque sean peligrosas (Chi cuadrado exacto < 0,001 en los tres). A pesar de que ambos grupos son consumidores de tabaco y alcohol, los que consumen alguna droga ilegal perciben un riesgo significativamente menor que el otro grupo en fumar un paquete de tabaco diario, tomar dos bebidas alcohólicas al día y tomar cuatro bebidas alcohólicas seguidas ( $p$ exacto $<0,001$ para el Chi cuadrado en los tres ítems), y también en el consumo regular de cannabis y el consu- 
mo menos regular de éxtasis y LSD ( $p$ exacto $<0,001$ para el Chi cuadrado en los tres consumos).

\section{Regresión logística de las variables relativas al riesgo sobre el consumo de drogas}

De todos estos ítems relativos al riesgo y asociados significativamente al consumo de alguna droga ilegal, el análisis de regresión logística seleccionó 8 que clasificaron correctamente a cuatro de cada cinco sujetos $(79 \%)$ en su grupo de consumo, $84 \%$ de los consumidores de alguna droga ilegal y $72 \%$ de los que consumen solo drogas legales. El factor de riesgo más relevante para ser un consumidor de alguna droga ilegal es el haber conducido bajo efectos de drogas ilegales (multiplica por 20 la probabilidad de ser consumidor), seguido de percibir un riesgo moderado o bajo en el consumo habitual de cannabis (multiplica por cuatro esta posibilidad), haber realizado alguna vez hurtos en establecimiento comerciales, percibir un riesgo moderado en el consumo semanal de éxtasis y haber realizado actos vandálicos. El factor protector más relevante es no haber conducido bajo efectos del alcohol, seguido de no haberse implicado nunca o recientemente en conductas orientadas a la búsqueda de sensaciones como hacer lo que le venga en gana o cosas locas aunque sean peligrosas.

\section{Predicción del consumo de alguna droga ilegal combinando los factores de riesgo y protección más relevantes}

Hasta este punto hemos descrito cómo diferentes dominios de factores de riesgo y protección funcionaban separadamente para predecir el consumo reciente de alguna droga ilegal en nuestra muestra. En este apartado (ver tabla 2) mostramos los resultados de un análisis basado en la regresión logística, realizado teniendo en cuenta los 23 mejores predictores del consumo de drogas ilegales encontrados en cada dominio o grupo de variables: sociodemográficas e historia de consumo de drogas, hábitos recreativos y variables relativas al riesgo. La ecuación resultante incluyó 11 de estos 23 predictores, y clasificó correctamente 9 de cada 10 consumidores recientes de alguna droga ilegal $(90 \%)$ y más de cuatro de cada cinco consumidores de drogas legales (83\%), un $87 \%$ de clasificación correcta global. La tabla 2 muestra (en su última columna) los predictores seleccionados y su nivel de significación relativa.

Nos parece interesante constatar que el dominio relativo a la vida recreativa es el que aporta mayor número de predictores a la ecuación final (6 de los 9 incluidos resultan seleccionados). Solamente la mitad de los predictores relativos al riesgo (4 variables) y uno de los 6 relativos a las características sociode- mográficas e historia de consumo de drogas resulta relevante en el modelo final. Las variables de la vida recreativa incluidas en el modelo final se refieren a los tres conceptos explorados: la implicación, el contexto cultural recreativo y su significado.

Dos factores de riesgo para el consumo reciente de alguna droga ilegal destacan claramente por su relevancia: el haber llegado a consumir cannabis (multiplica por 12 esta posibilidad en relación con los que no lo han llegado a consumir) y el haber consumido bajo los efectos de alguna droga ilegal (multiplica casi por 9 esta probabilidad). Otros factores de riesgo menos relevantes son (en orden decreciente): no percibir un alto riesgo en el consumo habitual de cannabis, y hacer desplazamientos largos para salir de marcha a divertirse. Los dos factores de protección más relevantes se refieren a la vida recreativa: no dar ninguna importancia a las drogas como motivo para salir de marcha ni para elegir locales donde se va a divertirse. Otros factores de protección son (en orden decreciente de relevancia) no haber conducido bajo efectos del alcohol, no haber realizado conductas orientadas a la búsqueda de sensaciones, tener como referencia culturas recreativas distintas a las "alternativas" o "after hour", no salir a divertirse durante los días laborables, y no dar una importancia máxima al escuchar música a la hora de salir a divertirse.

\section{DISCUSIÓN}

Se han considerado diferentes parámetros como indicadores de una progresiva "normalización" del consumo de drogas ${ }^{27}$ : disponibilidad y accesibilidad; tasas de prevalencia del consumo en el ciclo vital y consumo reciente; actitudes de acomodación hacia un consumo recreativo "sensible" de drogas, y grado de acomodación cultural al consumo de drogas ilegales. Teniendo en cuenta estos criterios, los resultados de nuestro estudio muestran una clara normalización del consumo de drogas legales e ilegales entre jóvenes que participan de la actividad nocturna de salir de marcha los fines de semana en diferentes ciudades Españolas.

No obstante, este consumo de drogas "normalizado" difiere substancialmente del perfil del consumidor recreativo y "no problemático" que postulan algunos autores $^{28}$. Encontramos una clara evidencia de abuso habitual del alcohol y tabaco, combinado con un uso relativamente frecuente de cannabis y de otras drogas en menor medida. También encontramos antecedentes de problemas por consumo de drogas, asociados a diversas conductas de riesgo para la salud y seguridad. Todos estos hallazgos corroboran la necesidad de diseñar intervenciones educativas y preventivas para 
Tabla 2: Predictores del consumo de drogas ilegales: Odds ratio, Intervalos de confianza al $95 \%$ y significación dentro de cada grupo de variables y globalmente.

\begin{tabular}{|c|c|c|}
\hline & En cada grupo de variables & Globalmente \\
\hline $\begin{array}{l}\text { Sociodemográficas e historia de consumo } \\
\text { Ha llegado a consumir cannabis } \\
\text { Edad de inicio en tabaco > } 15 \text { años } \\
\text { Varón } \\
\text { Se considera un mal estudiante } \\
\text { Vive con amigos, solo o en residencia } \\
\text { Ningún control familiar sobre el salir de marcha }\end{array}$ & $\begin{array}{c}52,9(28-99,9) * * * \\
0,51(0,38-0,67) * * * \\
1,5(1,1-2) * * \\
2(1,3-3) * * * \\
2,6(1,7-4) * * * \\
1,5(1,1-2,1) *\end{array}$ & $\begin{array}{c}12,18(26,3-56,9) * * * \\
- \\
- \\
- \\
- \\
-\end{array}$ \\
\hline $\begin{array}{l}\text { Hábitos recreativos } \\
\text { No sale habitualmente durante los días laborales } \\
\text { Hace habitualmente largos desplazamientos para salir } \\
\text { La sesión típica dura en promedio menos de } 8 \text { horas } \\
\text { Subcultura recreativa de adolescentes o normativos } \\
\text { Nunca va a fiestas "Rave" } \\
\text { Las drogas como razón para salir (nula importancia) } \\
\text { Escuchar música como razón para salir (importancia } \\
\text { moderada) } \\
\text { Bailar como motivo para salir (ninguna importancia) } \\
\text { Las drogas como razón para elegir lugares (ninguna } \\
\text { importancia) }\end{array}$ & $\begin{array}{c}0,46(0,33-0,65) * * * \\
1,74(1,2-2,5) * * \\
0,65(0,47-0,89) * * \\
0,48(0,35-0,66) * * * \\
0,59(0,40-0,86) * * \\
0,13(0-0,18) * * * \\
0,67(0,47-0,94) * \\
2,17(1,7-3,2) * * * \\
0,36(0,25-0,50) * * *\end{array}$ & $\begin{array}{c}0,56(0,38-0,83) * * \\
2(1,31-3,05) * * * \\
- \\
0,54(0,37-0,78) * * * \\
- \\
0,22(0,15-0,32) * * * \\
0,65(0,44-0,98) * \\
- \\
0,40(0,27-0,60) * * *\end{array}$ \\
\hline $\begin{array}{l}\text { Actitudes y conductas relativos al riesgo } \\
\text { No percibe un elevado riesgo en el consumo regular de } \\
\text { cannabis } \\
\text { No percibe un elevado riesgo en el consumo regular de } \\
\text { éxtasis } \\
\text { No ha conducido bajo los efectos del alcohol } \\
\text { Ha conducido bajo los efectos de drogas ilegales } \\
\text { Se ha implicado en conductas vandálicas alguna vez } \\
\text { Se ha implicado en hurtos en establecimientos } \\
\text { Nunca ha hecho lo que le ha venido en gana... } \\
\text { Nunca ha hecho cosas locas y peligrosas }\end{array}$ & $\begin{array}{c}4,15(2,6-6,7) * * * \\
1,9(1,3-2,8) * * * \\
0,32(0,21-0,48) * * * \\
20,79(12,7-34,1) * * * \\
1,61(1-2,5) * \\
2,1(1,5-3) * * * \\
0,61(0,42-0,91) * \\
0,52(0,36-0,74) * * *\end{array}$ & $\begin{array}{c}2,44(1,4-4,2) * * * \\
- \\
0,41(0,27-0,64) * * * \\
8,7(5,32-14,4) * * * \\
- \\
- \\
- \\
0,48(0,33-0,71) * * *\end{array}$ \\
\hline
\end{tabular}

${ }^{*}=\mathrm{p}<0,05 ;{ }^{*} \mathrm{P}<0,01 ;{ }^{* *} \mathrm{p}<0,001$. = no significativo.

estos consumidores de drogas que son usuarios de los espacios recreativos nocturnos del fin de semana.

Los resultados hallados en la predicción del consumo de alguna droga ilegal tampoco corroboran la existencia de un "efecto paradójico", como podría esperarse de un consumo de drogas normalizado y que se observa indistinta e independientemente de los factores de riesgo y protección individuales. La prevención paradójica se aplica cuando la mayoría de los sujetos con un comportamiento de riesgo se sitúan entre quienes no presentan los factores de riesgo validados (en este caso, para el consumo de drogas), recomendando de este modo un modelo de "prevención universal" 29 . En lugar de esto, nuestros resultados han mostrado una elevada capacidad de los factores de riesgo y protección intrapersonales para distinguir a los consumidores de drogas legales 
de los que han consumido recientemente (en el último mes) alguna droga ilegal además de drogas legales, a pesar de que muchos de los primeros también han llegado a consumir alguna droga ilegal en alguna ocasión. La capacidad para clasificar es generalmente algo mayor entre los consumidores recientes también de alguna droga ilegal, sugiriendo la necesidad de buscar otros factores que expliquen la resistencia o resiliencia ante el consumo de drogas ${ }^{30}$. De este modo, nuestros resultados muestran que el consumo combinado y reciente de drogas legales e ilegales es claramente distinguible del consumo solo de drogas legales, y que ofrece un perfil interpretable y teóricamente explicable.

Como hemos visto, el consume reciente de drogas ilegales en nuestra muestra es distinguible del consumo de drogas legales desde factores de riesgo y protección que tienen apoyo diverso en la literatura científica: Características sociodemográficas (género, emancipación tal vez temprana de la familia, etc.), variables relacionadas con el inicio más temprano en el consumo de drogas, baja adaptación escolar y otras conductas desviadas tempranas o recientes, conductas de riesgo pasadas o actuales, características de personalidad y variables mediadoras asociadas al riesgo y percepción del riesgo.

En este contexto, uno de los resultados más notables del estudio ha sido ilustrar cómo diversos indicadores relacionados con la vida recreativa nocturna del fin de semana alcanzan una elevada capacidad para distinguir quienes consumen solo drogas legales de quienes además han consumido recientemente alguna droga ilegal, tanto por separado como tenidas en cuenta conjuntamente con otros predictores intrapersonales validados científicamente. Estos factores de riesgo y protección en la vida recreativa se refieren a una alta dedicación e implicación en este tipo de ocio, un significado específico que va más allá de la mera diversión, socialización y fiesta e integra el consumo de drogas y al contexto físico y cultural donde se desarrolla.

¿Por qué estos componentes de la vida recreativa se han convertido en determinantes del consumo de drogas? Para muchos adolescentes y adultos jóvenes, cierto concepto de diversión se ha ido convirtiendo en dogma, una especia de experiencia casi-trascendental que canaliza y organiza su experiencia vital. De modo similar a otros ideales y valores humanos (amor, felicidad, etc.), la diversión es un estado que requiere determinadas condiciones para ser alcanzado. Muchos adolescentes y jóvenes salen de marcha ritualmente cada fin de semana, en una suerte de peregrinación por las áreas recreativas de las ciudades, tratando de encontrar una conexión con los espacios y encontrar la puerta que les da el tránsito desde un estado "normal" a otro estado "eufórico" y placentero.
Nuestros resultados sugieren la necesidad de planificar, implementar y evaluar actuaciones educativas y preventivas dirigidas al "salir de marcha" que tengan en cuenta el contexto físico y cultural, el significado de estas actividades recreativas y su gestión por los jóvenes. Existen ya iniciativas que proponen intervenciones ambientales más allá de la estrategia de reducción de riesgos y daños ${ }^{31}$. Estas estrategias podrían ser prometedoras si se benefician del estudio sistemático de los entornos recreativos en sus diferentes ámbitos (interpersonal, cultural y físico) y niveles de estudio (macro, meso y micro) ${ }^{32}, 33$. La prevención el uso y abuso de drogas en los espacios recreativos requiere también comprender, decodificar y de-construir los procesos y resultados que llevan a un significado de la diversión asociado a consumo de drogas y riesgos diversos para la salud y seguridad.

Es necesario tener en cuenta algunas limitaciones del estudio. Su naturaleza transversal limita la utilidad predictiva de los factores de riesgo y protección identificados para el consumo reciente de drogas ilegales. Los procedimientos de muestreo no garantizan que las sub-muestras obtenidas en cada ciudad sean representativas de los usuarios de los espacios recreativos estudiados. No obstante, podemos considerar estas sub-muestras como una buena representación de las sub-culturas recreativas predominantes en estas ciudades, como era el objetivo del muestreo. Teniendo en cuenta estas limitaciones, los hallazgos del estudio pueden guiar el estudio más profundo de los componentes de la vida recreativa que determinan el consumo de drogas en espacios recreativos, junto con otros factores de riesgo y protección intrapersonales e interpersonales validados científicamente.

\section{RECONOCIMIENTOS}

Este estudio se llevó a cabo con una ayuda concedida a Irefrea por la DGPNSD en la convocatoria 19981999.

\section{REFERENCIAS}

1. European Monitoring Centre for Drugs and Drug Addiction. Annual report 2003: the state of the drugs problem in the European Union and Norway. Annual report 2003: the state of the drugs problem in the European Union and Norway 2003

2. European Monitoring Centre for Drugs and Drug Addiction. Annual report 2004: the state of the drugs 
problem in the European Union and Norway. European Monitoring Centre for Drugs and Drug Addiction 2004

3. United Nations. World situation with regard to drug abuse. Commission on Narcotic Drugs, Fortyseven session. Vienna 15-22 M2, editors. 2004. Ref Type: Unpublished Work

4. Skara S, Sussman S. A review of 25 long-term adolescent tobacco and other drug use prevention program evaluations. Prev Med 2003; 37: 451-74.

5. Becoña E. Bases teóricas que sustentan los programas de prevención de drogas. Madrid: Plan Nacional Sobre Drogas; 1999.

6. Becoña E. Bases científicas de la prevención de las drogodependencias. Madrid: Plan Nacional Sobre Drogas; 2002.

7. Brown RT. Risk factors for substance abuse in adolescents. Pediatr Clin North Am 2002; 49:247-55.

8. Kendler KS, Jacobson KC, Prescott CA, Neale MC. Specificity of genetic and environmental risk factors for use and abuse/dependence of cannabis, cocaine, hallucinogens, sedatives, stimulants, and opiates in male twins. Am J Psychiatry 2003 Apr; 160:687-95.

9. Muñoz-Rivas MJ, Graña JL, Cruzado JA. Factores de riesgo en drogodependencias: Consumo de drogas en adolescentes. Madrid: Paradox Libros; 2000.

10. Arthur MW, Hawkins JD, Pollard JA, Catalano RF, Baglioni AJ Jr. Measuring risk and protective factors for substance use, delinquency, and other adolescent problem behaviors. The Communities That Care Youth Survey. Eval Rev 2002; 26: 575-601.

11. Rhodes T, Lilly R, Fernandez C, Kemmesis UE, Ossebaard HC, Lalam N, et al. Risk Factors Associated With Drug Use: the importance of "risk environment". Drugs: education, prevention and policy 2003; 10: 303-29.

12. Becoña E. Adolescentes y el consumo de drogas. Pap Psicol 2000; 77 (25-32).

13. Calafat A, Bohrn K, Juan M, Kokkevi A, Maalsté N, Mendes $F$, et al. Night life in Europe and recreative drug use. SONAR 98. Palma de Mallorca: IREFREA; 1999.

14. Calafat A, Stocco P, Mendes F, Simon J, van de Wiijngaart G, Sureda MP, et al. Characteristics and social representation of ecstasy in Europe. Palma de Mallorca: IREFREA; 1998.

15. Calafat A, Bohrn K, Juan M, Kokkevi A, Maalsté N, Mendes $F$, et al. Night life in Europe and recreative drug use. SONAR 98. Palma de Mallorca: IREFREA; 1999.

16. Calafat A, Fernández $C$, Juan M, Bellis MA, Bohrn K, Hakkarainen $\mathrm{P}$, et al. Risk and control in the recreational drug culture. SONAR PROJECT. Palma de Mallorca: IREFREA; 2001.

17. Calafat $A$, Fernández $C$, Juan $M$, Anttila $A H$, Arias $R$, Bellis $M A$, et al. Enjoying the nightlife in Europe. The role of moderation. Palma de Mallorca: IREFREA; 2003.
18. Calafat A, Fernández $C$, Juan $M$, Anttila AH, Bellis MA, Bohrn K, et al. Cultural Mediators in a Hegemonic Nightlife. Palma de Mallorca: IREFREA; 2004.

19. Calafat A, Juan M, Becoña E, Fernández C, Gil E, Palmer $A$, et al. Salir de marcha y consumo de drogas. Madrid: Plan Nacional Sobre Drogas; 2000.

20. Fendrich M, Wislar JS, Johnson TP, Hubbell A. A contextual profile of club drug use among adults in Chicago. Addiction 2003 Dec; 98:1693-703.

21. Wadsworth EJ, Moss SC, Simpson SA, Smith AP. Factors associated with recreational drug use. J Psychopharmacol 2004; 18: 238-48.

22. Galliot-Guilley M. Synthetic drugs consumed at "rave parties". Ann Pharm Fr 2004; 62: 158-64.

23. Riley SC, James C, Gregory D, Dingle H, Cadger M. Patterns of recreational drug use at dance events in Edinburgh, Scotland. Addiction 2001; 96:1035-47.

24. Rome ES. It's a rave new world: rave culture and illicit drug use in the young. Cleve Clin J Med 2001; 68: 54150 .

25. Adlaf EM, Smart RG. Party subculture or dens of doom? An epidemiological study of rave attendance and drug use patterns among adolescent students. J Psychoactive Drugs 1997; 29: 193-8.

26. Fendrich $M$, Wislar JS, Johnson TP, Hubbell A. A contextual profile of club drug use among adults in Chicago. Addiction 2003 Dec. 98; 12:1693-703.

27. Parker $H$, Williams L, Aldridge J. The Normalization of "Sensible" Recreational Drug Use: Further Evidence from the North West England Longitudinal Study. Sociology 2002; 36: 941-64.

28. Nicholson T, White J, Funcan DF. A Survey of adult recreational drug use via the world wide web: The DRUGNET study. J Psychoactive Drugs 1999; 31: 41522.

29. Stockwell T, Toumbourou JW, Letcher P, Smart D, Sanson A, Bond L. Risk and protection factors for different intensities of adolescent substance use: when does the Prevention Paradox apply? Drug Alcohol Rev 2004 Mar; 23: 67-77.

30. Fergus S, Zimmerman MA. Adolescent Resilience: A Framework for Understanding Healthy Development in the Face of Risk. Annu Rev Public Health. In press 2005.

31. Bellis MA, Hughes $K$, Lowey $H$. Healthy nightclubs and recreational substance use. From a harm minimisation to a healthy settings approach. Addict Behav 2002 Nov; 27:1025-35.

32. Davis JR, Tunks E. Environments and addiction: A proposed taxonomy. Int J Addictions 1991; 25 (7A\&8A):805-26.

33. Gilford R, Hine DW. Substance use and the physical environment: The early action on a newly completed field. Int J Addictions 1991; 7A\&8A:827-53. 
